\title{
Prognostic Factors in Soft Tissue Sarcoma
}

\author{
Luiz Eduardo Moreira Teixeira, \\ Jose Carlos Vilela and Ivana Duval De Araujo \\ Federal University of Minas Gerais \\ Brazil
}

\section{Introduction}

Soft tissue sarcoma (STS) represents a heterogeneous group of mesenchymal malignant tumors with variable natural history. This term was first introduced to describe a circumscribed neoplasm consisting of malignant fat cell occurring principally in the trunk (Abernethy, 1817). Nowadays, more than 30 types of STS have been described, and many of these present innumerable subtypes. Despite this wide range of entities, they represent less than $1 \%$ of all types of cancer, but still one of the most therapeutically challenging group of tumor (Choong \& Rudiger, 2008). Surgery is the keystone of treatment, associated or not to adjuvant method, and the two aims are avoid metastatic spread and local relapse.

Despite advances in local control of the sarcomas, the metastatic disease, which is the cause of death for most patients, presents little recent advances. In fact, $10 \%$ of patients have metastasis at the diagnosis and $25 \%$ with localized disease will develop distant spread (Delaney et al, 1991). The systemic disease occurs primarily by hematogenic spread, and the lungs are the most common distant organs involved by the distant metastasis. Some subtypes demonstrated predilection for lymphatic route of dissemination such as synovial sarcoma and epithelioid sarcoma while the alveolar sarcoma targets the brain involvement.

In contrast to bone sarcomas, soft tissue malignancies do not respond well to chemotherapies schemes. This may be explained by the diversities of subgroups or by different factors that affect the prognosis. Therefore, the difficult in treating soft tissue sarcomas is determinate which patients will need the adjuvant chemotherapy or radiation therapy. The indication of these methods is guided by factors that predict the risk of metastatic disease or local recurrence. These factors are called prognostic factors and the purpose of this chapter is to define the different prognostic factors of soft tissue sarcoma.

As a heterogeneous group, the behavior and natural evolution of this tumor are variable, but, in general aspects the metastatic spreading is observed in about one third of the patients, local relapse is seen in $10 \%$ to $30 \%$ and the global 5 -year survival is $60 \%$. Different factors are reported in the literature such as tumor grade, size, location, vascular invasion, histological necrosis and presence of cytogenetic markers. However, the importance of each factor is unclear. In this chapter we will discuss each prognostic factor including: age, sex, location, size, subtype, histological aspects, grade, surgical manipulation, recurrence, imunohistochemical pattern, genetic and gene markers. 
In this chapter the prognostic factors can be separated into three categories: patient factors, tumor factors and treatment factors.

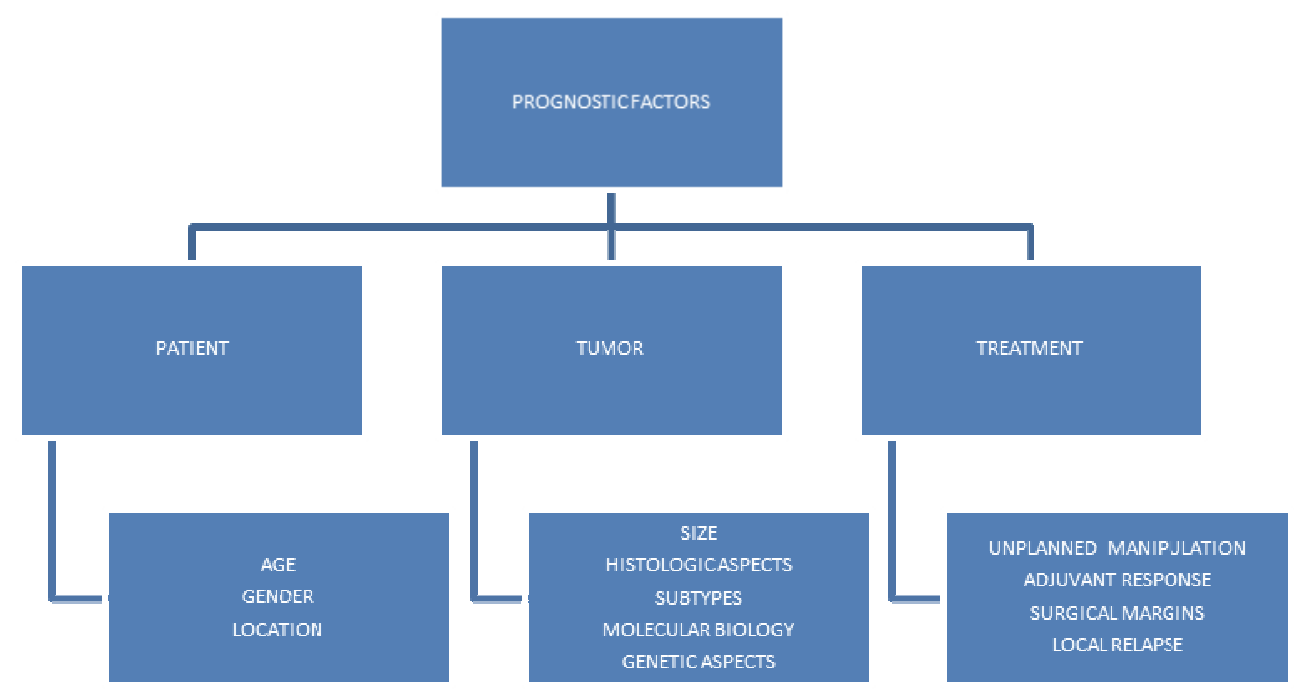

Fig. 1. Resume of prognostic factor of soft tissue Sarcoma

\section{Prognostic factors}

\subsection{Patient factors}

The factors in the literature related to patients are: age, gender and tumor localization. In some studies, patients older than 50 years and male are associated with a bad prognostic (Gustafson et al. , 1994). Nevertheless, these factors have not been proven of value in predicting the risk for local recurrence and metastasis. Although the shorter survival observed in the elderly, this seems to be related to tumor size and aggressiveness in this age group 35, 36. Even if gender and age had some importance, this seems to be a minor one. (Teixeira et al., 2009)

Localization has also been evaluated as a prognostic factor, according to the segment involved, depth or the site related to the anatomical compartments. The depth in relation to the muscle fascia is considered a relevant factor for the prognosis, being included in some prognostic systems as the AJCC/UICC. However, some studies doubt the validity of depth as an isolated factor of worse prognosis, they have found the same survival rate when the tumors are adjusted by the histological malignancy and size (Rydholm \& Gustafson, 2003). 
The extra compartmental localization also seems to be associated with a bad prognostic. The tumors considered extra compartmental usually present more difficult surgical treatment and thinner margins, provided that they are in close contact to neurovascular structures. Both, the difficult to obtain proper margins and the fact that they are situated in areas with rich vascularization and lymphatic drainage help to systemic disseminate and local recur, which may explain the results obtained by some studies. (Teixeira et al., 2009)

More proximal tumors, situated in the scapular or pelvic girdle, in general present delayed diagnosis and a bigger size, thus a worse prognosis. On the other side, more distal tumors present longer survival rate, but many times they require amputations in order to achieve the desired surgical margin. Some studies pointed out a survival rate of $47 \%$, with a local recurrence of $35 \%$ in tumors of the gluteus area, while tumors in hands and feet present a survival rate between $80 \%$ and $82 \%$ in five years and a local recurrence rate between $17 \%$ and $21 \%$ (Behranwala et al., 2004). Despite the disparity in the survival and local recurrence rates, the risk does not seem to be associated with the localization but with the size of the tumor in the moment of the diagnostic, as long as distal tumors tend to present earlier and smaller.

\subsection{Tumor's factors}

The factors associated with tumors seem to be the most important ones to determine the prognostic of systemic spread. Among them, we call special attention for size, histological and genetic aspects and the histological subtypes.

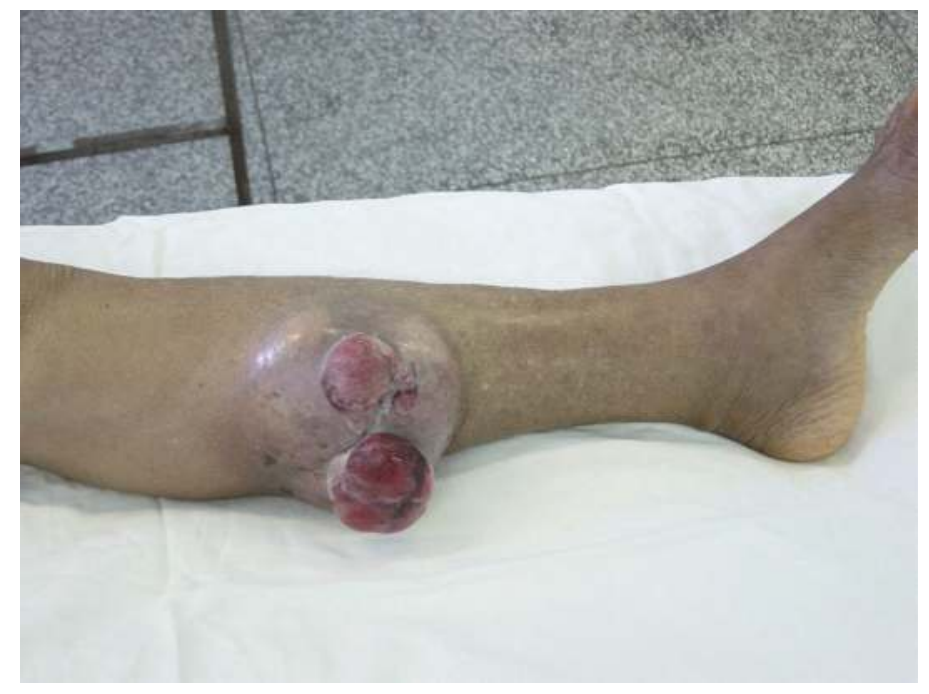

Fig. 2. Large tumors representing an adverse effect in survival 
The size of the tumor is implicated as an important factor for systemic disease and local recurrence. However, there is no consensus determining the cutoff for groups at high risk. Some clinical trials use $5 \mathrm{~cm}, 8 \mathrm{~cm}$ or $10 \mathrm{~cm}$ as the cutoff. In general, the growth of $5 \mathrm{~cm}$ in the tumor size increases the risk for metastasis 1.5 times (Trovik et al., 1994). Provided that, the tumors bigger than $5 \mathrm{~cm}$ should been considered as possessing a worse prognostic.

The histologic grading is a method for stratifying STS into groups of different prognosis based on histological aspects such as cellularity, atypia, mitotic index, necrosis, pleomorphism and histotype. Most of studies show that high grade of malignancy possess a strong influence in developing metastasis and local recurrence, being one of the most important independent factors related to the aggressiveness of the STS. However, the lack of universal classification, the inaccuracy of each histological component, the examiner subjectivity and the different grades of aggressiveness of each histological subtypes reduce the reproducibility. The combination of these factors creates several grading systems and prognostic models. Some schemes, such as that of the American Joint Committee on Cancer (AJCC), have also included nodal involvement. The most widely used grading systems include those proposed by the French Federation Nationale des Centres de Lutte Contre le Cancer (FNCLCC) and the National Cancer Institute (NCI), the staging system established by the AJCC, and a sarcoma-specific mortality nomogram developed at the Memorial Sloan-Kettering Cancer Center (MSKCC). Despite of this, the high-grade histologic aspect of the tumor must be considered one of most determinant factor in overall survival.

Among the specific histologic aspects, vascular invasion and necrosis seem the most important one as an isolated prognostic factor. Vascular invasion represents a wellestablished prognostic factor in several tumor types, including malignant melanoma, papillary thyroid cancer, endometrial cancer, and testicular cancer. In STS, vascular invasion has repeatedly shown prognostic value, but it is generally not applied systematically in pathological evaluations. Vascular invasion has been identified with variable prevalence in different studies, which may hamper its clinical application. Tumor necrosis has repeatedly been established as a negative prognostic factor in STS.23,30-34 It also represents an important factor in the FNCLCC and the NCI grading systems, which apply cutoff values of 15\% (microscopic evaluation) and 50\% (macroscopic evaluation), respectively. A more recent histologic find used in the STS grading is the pattern of growth. Growth pattern was classified as pushing or infiltrative, irrespective of the extent of infiltration. Soft tissue tumors with microscopically infiltrative pattern of growing have a significantly higher risk for local and systemic recurrence compared with a "pushing" growth pattern.

The histologic subtypes was previously not considered a important factor in the prognosis of patients with STS, but the differences in response to adjuvant treatments improve the value of the histotypes in predicting metastatic spreading. Some subtypes have a high risk to develop distant disease such as synovial sarcoma, epithelioid sarcoma, rhabdomyosarcoma and soft tissue Ewing`s sarcoma

\subsubsection{Genetics and molecular aspects}

Prognostication for adults with STS is primarily based on tumor size, histologic grade, depth, location, histologic subtype, and the presence or absence of distant metastasis. However, recent studies suggest that proliferation markers may improve the prognostic 
value of standard clinical-pathologic markers. These studies evaluated the DNA activity, antigen markers and oncogenes. The first studies have shown that tumor cell proliferative activity and/or DNA content are related to the outcome of patients with STS. Several methods have been used to assess cell proliferation, including enumeration of mitotic figures, thymidine labeling indices, DNA-flow cytometric analysis, and immunohistochemical analysis of proteins associated with proliferation. Furthermore, multivariate analysis revealed that DNA content was an independent prognostic indicator. The widespread availability of DNA analysis by flow cytometry and image analysis, as well as the magnitude of the difference in predicting survival, suggest that DNA analysis may be useful clinically, for example in selecting candidates for adjuvant systemic therapy. However, such statement will require further study (Levine, 1999). .

The second aspect is histological markers. The most studied is Ki-67, an antigen described in 1983 as a human nuclear antigen associated with cellular proliferation. Ki-67 expression has been found related to other proliferation markers and to p53 mutations. To date, the threshold value of immunohistochemical staining for Ki-67 expression that defines a highrisk lesion for Ki-67 expression is unclear, but is observed in $40 \%$ as a "positive" level of expression. Although his prognostic significance is unclear, it has been associated with prognosis (Ueda et al. 1989).

The third aspect is the effect of oncogenes. The oncogenes most studied are p53, ras gene family, myc, and the RB1.

The p53 gene, located on human chromosome 17p13, encodes a nuclear phosphoprotein involved in transcriptional regulation, translational control, DNA repair, cell cycle control, cell differentiation, and apoptosis. The p53 gene is the most commonly mutated gene in human solid tumors (Bartek et al., 1991), its mutant expression is observed in about $61 \%$ of patients with STS (Wadayama et al., 1993) and is associated with poor prognosis for distant spread, disease free-survival and drug resistance. Although its clinical validity is unclear.

The ras gene family consists of three genes located on chromosomes 1,11, and 12 . Several small series of STS have shown low incidence of ras mutations in the range of $3 \%$ to $35 \%$. Although ras mutations are demonstrable in some types of STS, it is doubtful that this represents the original stimulation for malignant transformation or a clinically useful prognostic marker.

The myc oncogene encodes the production of a nuclear phosphoprotein, which binds to DNA and can transform cells both in vitro and in vivo [50]. This gene is normally a single copy on chromosome 2. Amplification myc has been shown to be a poor prognostic indicator for children with neuroblastoma. Several reports have been published of myc expression in STS (Dias et al., 1990). In a study of rhabdomyosarcomas, another report suggested that myc amplification is limited to the alveolar subtype. A study of 23 STS cases found myc amplification in 30\% and suggested it was correlated with higher grade and poorer survival [54]. Although the data for myc expression/ amplification suggest a putative relationship between myc with progression and survival, confirmatory studies are needed before validating myc as a prognostic factor in routine clinical practice (Barrios et al., 1994).

The c-erbB2 is located on human chromosome17 and encodes a transmembrane protein (p185) which has remarkable homology with the epidermal growth factor receptor (EGFR). 
Few reports have analyzed c-erbB2 amplifications or overexpression in STS. Amplification in STS seems to be unusual, occurring in only six of 105 cases in one report (. No studies have attached clinical significance to c-erbB2 amplification and/or overexpression. Furthermore, the low rates of c-erbB2 alterations do not suggest a role in the initiation or progression of STS.

The human retinoblastoma gene, RB1, located on chromosome 13, is another tumor suppressor gene involved in the genesis of STS. Germline's mutations of RB1 result in the hereditary form of retinoblastoma. In addition, somatic mutations of this gene and/or absent p110RB protein expression have been observed in various human tumors and tumor cell lines. Alterations in RB1 or p110RB protein expression have been observed in up to $70 \%$ of the STS studied, but do not appear to consistently have clinical prognostic value (Karpeh et al.,1995).

\subsection{Treatment factor}

Although STSs do not have a good response as for bone sarcomas, some subtypes can be treated with chemotherapy schemes. Systemic chemotherapy is considered the only therapeutic option for patients presenting with widely metastatic disease or with locally advanced disease not amenable to surgery or radiotherapy. For the majority of these patients, cytotoxic chemotherapy should be regarded as palliative, although in a small subset of patients long-term survival may be achieved. The standard chemotherapy is doxorubicin-based therapy, but others subtypes have a good response to ifosfamide like synovial sarcoma. Rhabdomyosarcoma, soft tissue Ewing`s sarcoma and others round cell soft tissue tumors have good response to this adjuvant treatment. So the response to the treatment can be used as a prognostic factor, but the clinical use is still unclear.

Other question concerning the influence of the treatment in the prognosis is the previous manipulation of the tumor by an unplanned resection. Many times patient and the assistant physician came across a diagnosis of an unsuspected sarcoma after a lump resection. This manipulation can spread cell tumor. We consider that the clinical problems following an unplanned resection of a sarcoma are more dependent of the technical difficulties concerning the oncological surgery principles when reoperating the patient than the malignance of the tumor itself. If the salvage treatment could be performed appropriately, the oncological outcomes may not be influenced.

Another topic is the effects of a local recurrence in the survival and metastatic disease. There is little doubt in the literature that local relapse is associated with a worse prognosis. The unsolved issue is whether local relapse is causative. The occurrence of local relapse per se might favor the systemic spread of disease and, therefore, directly affect survival, or it might simply be a marker of biological tumor aggressiveness. Theoretically, the two mechanisms may coexist and possibly interact, with both contributing to the outcome. Some patients had a local relapse after surgery performed at nonreferral centers, with unplanned resections with marginal or intralesional margins. Others, relapse even with wide margins and planned procedure. In the last cases, the recurrence suggests an aggressive sarcoma and probably is associated with worse prognosis. If tumor aggressiveness could be objectively assessed initially, a different surgical policy might be justified from the very beginning (Gronchi et al., 2007). 


\section{Conclusion}

Soft tissue sarcomas have many factors that can influence the overall survival of the patients. These factors are related to the patients, tumor and treatment. To achieve the best possible result, the physician must take all of them in consideration and try to control the factors that have influence in the outcome and are possible to be handled to minimize the effects of the others witch he can't modify.

\section{References}

Albernethy J. Surgical Aberrations. J Surgical Aberrations 1817, 2, 17 - 30.

Barrios C, Castresana JS, Kriecbergs A: Clinicopathologic correlations and short-term prognosis in musculoskeletal sarcoma with cmyc oncogene amplification. Am J Clin Oncol 1994;17:273-276.

Bartek J, Bartkova J, Vojtesek B, et al: Aberrant expression of the p53 oncoprotein is a common feature of a wide spectrum of human malignancies. Oncogene 1991;6:1699-1703.

Behranwala KA, A`Hern R, Omar A, Thomas M. Prognosis of lymph node metastasis in soft tissue sarcoma. Ann Surg Oncol 2004, 11(7): 714 - 719.

Choong PFM, Rudiguer HA. Prognostic fator in soft tissue sarcoma: what have we learned? Expert Rev Anticancer Ther, 8(2): 139-146, 2008.

Delaney T, Yang J, Glatstein E. Adjuvant Therapies for adult patients with soft tissue sarcoma. Oncology 5, 105-118, 1991.

Dias P, Kumar P, Marsden HB, et al: N-myc gene is amplified in alveolar rhabdomyosarcomas (RMS) but not in embryonal RMS. Int J Cancer 1990;45:593596.

Gustafson P, Dreinhofer K, Ryldhom A. Soft tissue sarcoma should be treated at a tumor center. A comparison of quality of surgery in 375 patients. Acta Orthop Scand. 1994; 65: 47-50.

Karpeh MS, Brennan MF, Cance WG, et al: Altered patterns of retinoblastoma gene product expression in adult soft-tissue sarcomas. Br J Cancer 1995;72:986-991.

Levine EA. Prognostic Factors in Soft Tissue Sarcoma. Semin. Surg. Oncol. 17:23-32, 1999.

Rydholm A and Gustafson P. Should tumor depth be included in prognostication of soft tissue sarcoma? BMC Cancer 2003, 3:17 (http: //www.biomedcentral.com/14712407/3/17).

Teixeira LE, Araújo ID, de Andrade MA, Gomes RA, Salles PG, Ghedini DF. Local recurrence in soft tissue sarcoma: prognostic factors. Rev Col Bras Cir 2009, 36(5):377-81.

Trovik CS, Bauer HC Local recurrence of soft tissue sarcoma a risk factor for late metastases. 379 patients followed for 0.5 - 20 years. Acta Orthop Scand 1994, 65: 553 -558.

Uda RB, Cundiff D, August CZ, et al: Growth factor receptor andrelated oncogene determination in mesenchymal tumors. Cancer1993;71:3526-3530.

Ueda T, Aozasa K, Tsujimoto M, et al: Prognostic significance of Ki-67 reactivity in soft tissue sarcomas. Cancer 1989;63:1607-1611. 
Wadayama B, Toguchida J, Yamaguchi T, et al: p53 expression and its relationship to DNA alterations in bone and soft tissue sarcomas. Br J Cancer 1993;68:1134-1139. 


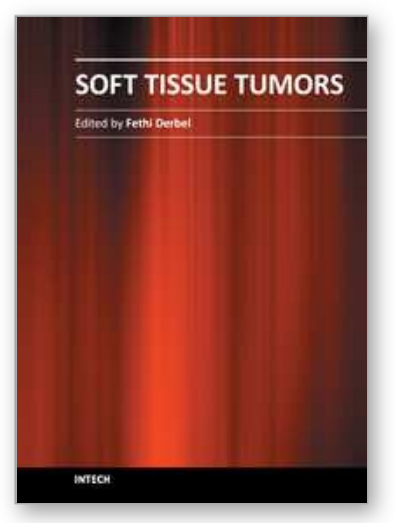

\author{
Soft Tissue Tumors \\ Edited by Prof. Fethi Derbel
}

ISBN 978-953-307-862-5

Hard cover, 270 pages

Publisher InTech

Published online 16, November, 2011

Published in print edition November, 2011

Soft tissue tumors include a heterogeneous group of diagnostic entities, most of them benign in nature and behavior. Malignant entities, soft tissue sarcomas, are rare tumors that account for $1 \%$ of all malignancies. These are predominantly tumors of adults, but $15 \%$ arise in children and adolescents. The wide biological diversity of soft tissue tumors, combined with their high incidence and potential morbidity and mortality represent challenges to contemporary researches, both at the level of basic and clinical science. Determining whether a soft tissue mass is benign or malignant is vital for appropriate management. This book is the result of collaboration between several authors, experts in their fields; they succeeded in translating the complexity of soft tissue tumors and the diversity in the diagnosis and management of these tumors.

\title{
How to reference
}

In order to correctly reference this scholarly work, feel free to copy and paste the following:

Luiz Eduardo Moreira Teixeira, Jose Carlos Vilela and Ivana Duval De Araujo (2011). Prognostic Factors in Soft Tissue Sarcoma, Soft Tissue Tumors, Prof. Fethi Derbel (Ed.), ISBN: 978-953-307-862-5, InTech, Available from: http://www.intechopen.com/books/soft-tissue-tumors/prognostic-factors-in-soft-tissue-sarcoma

\section{INTECH}

open science | open minds

\section{InTech Europe}

University Campus STeP Ri Slavka Krautzeka 83/A 51000 Rijeka, Croatia Phone: +385 (51) 770447

Fax: +385 (51) 686166 www.intechopen.com

\section{InTech China}

Unit 405, Office Block, Hotel Equatorial Shanghai No.65, Yan An Road (West), Shanghai, 200040, China 中国上海市延安西路65号上海国际贵都大饭店办公楼 405 单元 Phone: +86-21-62489820

Fax: $+86-21-62489821$ 
(C) 2011 The Author(s). Licensee IntechOpen. This is an open access article distributed under the terms of the Creative Commons Attribution 3.0 License, which permits unrestricted use, distribution, and reproduction in any medium, provided the original work is properly cited. 